\title{
Formulation and Evaluation of Herbal Anti-Dandruff Shampoo from Bhringraj Leaves
}

\author{
P. Vinod Kumar ${ }^{* 1}$, P. Venkates wara Rao ${ }^{1}$, R.Prince ${ }^{1}$, K.Tere jamma ${ }^{1}$, T.Chaitanya ${ }^{1}$, Prasanna \\ Kumar Desu ${ }^{1}$ \\ ${ }^{*}$ Department of Pharmacy, St. Mary's Group of Institutions Guntur, Chebrolu (V\&M), Guntur, Andhra \\ Pradesh, India.
}

\author{
*Corresponding Author: P. Vinod Kumar, Department of Pharmacy, St. Mary's Group of Institutions \\ Guntur, Chebrolu(V\&M), Guntur, Andhra Pradesh, India.
}

\begin{abstract}
Abs tract: Dandruffis a common disorder affecting the scalp condition caused by yeast Pityrosporum. Dandruff cannot be completely eliminated but can only be managed and effectively controlled. A shampoo is a preparation containing surfactant (i.e. surface active material) in a suitable form - liquid, solid or powderwhich when used under the specified conditions will remove surface grease, dirt, and skin debris from the hair shaft and scalp without adversely affecting the user. Various anti-fungal agents are employed in hair care preparations for the treatment dandruff. These products show many side effects like loss of hair, increased scaling, itching, irritation, nausea, and headache. Hence an attempt was made to formulate herbal antidandruff shampoo which is effective in terms of safety and treating the dandruff condition better than the chemical based anti-dandruff shampoo. Herbal anti-dandruff shampoos were formulated using herbal based ingredients like Bhringraj Leaves extract, Lemon Grass Oil, Neem oil, Henna, Aloe Vera gel and other ingredients for preparing base shampoo. The formulated shampoos were subjected to evaluation parameters like visual inspection, $\mathrm{pH}$, viscosity, Percentage of solids contents, Dirt dispersion, Surface tension, Foaming ability and foam stability, anti-fungal activity test using Pityrosporum Ovale strain. Formulation (F4) exhibited good antifungal activity i.e., maximum zone of inhibition. Hence it was subjected to safety studies on animals, such as eye irritation test and skin sensitivity test.
\end{abstract}

Keywor ds: Dandruff, herbal anti-dandruffshampoo, Bhringraj Leaves extract and Foaming Ability.

\section{INTRODUCTION}

A shampoo may be defined as a preparation of a surfactant (i.e. Surface active material) in a suitable form-liquid, solid, or powder which when used under the conditions specified will remove surface grease, dirt and skin debris from the hair, shaft and scalp without affecting adversely the hair, scalp or the health of the user. The word shampoo in English usage dates back to 1762, with the meaning "to massage". The word derived from Anglo-Indian shampoo, in turn from Hindi champoo imerative of champna to smear, knead the muscles, massage. Today, a plethora of shampoos are available for men and women.

A good shampoo should almost immediately form abundant foam irrespective of the type of water used or the nature of soil or fat to be removed from hair. Though foam formation is not released to the cleansing effect, but people psychologically always prefer a high foam product. Some good shampoos are found to have side effects like drying effect on the hair. This leaves the hair too dry to handle or comb. So proper conditioning of the hair is also an important consideration, some shampoos cause irritation to the eye and a lasting corneal cloud. These should be avoided. The functions of shampoo are expected to be various. A good and acceptable shampoo should have the following characteristics ${ }^{1}$.

\subsection{Composition of Shampoos 2-6}

The following are the ingredients used for preparation of shampoos. They include Primary surfactants e.g. Sodium lauryl sulphate, triethanol lauryl sulphate. Secondary surfactants e.g. dialkyl sulphosuccinates, monoalkyl sulphosuccinates. Germicides and Antidandruff agent's e.g. salicylic acid, Benzoic acid. Conditioning agents e.g. Fatty substances like lanolin, oils. Pearlescent agent's e.g.4methyl-7-diethylamino coumarin. Sequestrates e.g. Sodium salt of EDTA. Thickening agents e.g. Alginates. Preservatives e.g. formaldehyde, methyl paraben, propyl paraben. Solubilizing agents' e.g. aliphatic alcohols, Urea etc. 
Dandruff7 represents one of the most common dermatological skin conditions and is a chronic, noninflammatory condition of the scalp that is characterized by excessive scaling of scalp tissue. Dandruff is apparently caused by a fungus called Malassezia restricta and M. globosa. Malassezia formerly called Pityrosporum is a yeast causing infection of skin and scalp. It often causes itching. Warm and humid atmosphere, overcrowding and poor personal hygiene are ideally suited for the growth of Malassezia 8 . Dandruff affects 5\% of the population and mostly occurs after puberty, between 20-30 years and dandruff affects males more than females. Dandruff occurs exclusively on skin in areas with high levels of sebum. Symptoms of dandruff mainly include itching, flakes; redness of scalp. Dandruff can be treated in two ways, by using herbal based antidandruff shampoo and by using chemical based antidandruff shampoo. A shampoo is a preparation containing surfactant (i.e. surface active material) in a suitable form - liquid, solid or powder - which when used under the specified conditions will remove surface grease, dirt, and skin debris from the hair shaft and scalp9 without adversely affecting the user. Most shampoos contain water, a detergent (cleaning agent), surfactant (lather making agent), salt, fragrance (natural and artificial), preservative and food coloring. With the exception of water and salt (sodium chloride), different chemical compounds are used depending on the desired result of the shampoo. Many shampoos also contain vitamins and moisturizing alcohols to prevent too much of the hair and scalp's natural oils from being stripped away during cleansing. Herbal Cosmetics, here in after referred as Products, and are formulated, using various permissible cosmetic ingredients to form the base in which one or more herbal ingredients are used to provide defined cosmetic benefits only, shall be called as "Herbal Cosmetics10-11". Herbal drugs or their formulations are viable alternative to synthetic drugs. During the past few decades, there has been a dramatic increase in the use of natural products in cosmetics. Now-a-days, many herbal shampoos are available in the market which contains herbal ingredients such as plant extracts and essential oils. There are large number of plants which are reported to have beneficial effects on hair and are commonly used in shampoos. Many herbs such as Hibiscus rosasinensis, Lawsonia inermis, Citrus aurantifolia, Phyllanthus emblica, Ocimum sanctum, Zingiber officinalis, Azadirecta indica, aloe vera have been used as an anti-dandruff agents in many shampoo formulations.

\section{Materials AND Methods}

Lemon Grass Oil, Neem Oil, Bhringraj Powder, Henna oil was procured from local market. Sodium lauryl sulfate glycerin, EDTA was procured form SVR Labs, Hyderabad.

\subsection{Pre paration of Shampoo}

Preparation of anti-dandruff shampoo: Shampoo was formulated using simple mixing process. Herbal anti-dandruff shampoo was formulated by adding the required amounts of herbal ingredients as given in the formulation table no 1

Table1. Formulation of Herbal Anti-Dandruff Shampoo

\begin{tabular}{|c|c|c|c|c|c|}
\hline & F1 & F2 & F3 & F4 & F5 \\
\hline Neem oil & 0.5 & 1.0 & 1.5 & 2.0 & 2.5 \\
\hline Lemon Grass Oil & 0.5 & 1.0 & 1.5 & 2.0 & 2.5 \\
\hline Bhringraj Powder & 0.5 & 1.0 & 1.5 & 2.0 & 2.5 \\
\hline Henna Oil (ml) & 0.5 & 1.0 & 1.5 & 2.0 & 2.5 \\
\hline Sodium Lauryl Sulfate(gms) & 20 & 15 & 10 & 5 & - \\
\hline Glycerin (ml) & 1 & 1 & 1 & 1 & 1 \\
\hline EDTA (gm.) & 0.15 & 0.15 & 0.15 & 0.15 & 0.15 \\
\hline Sodium Hydroxide & To adjust pH & To adjust pH & To adjust pH & To adjust pH & To adjust pH \\
\hline Water & q.s & q.s & q.s & q.s & q.s \\
\hline Perfume & q.s & q.s & q.s & q.s & q.s \\
\hline Total & $100 \mathrm{ml}$ & $100 \mathrm{ml}$ & $100 \mathrm{ml}$ & $100 \mathrm{ml}$ & $100 \mathrm{ml}$ \\
\hline
\end{tabular}

\subsection{Evaluation Parameters}

To evaluate the prepared formulations, quality control tests including visual assessment and physicochemical controls such as $\mathrm{pH}$, density and viscosity were performed. Also, to assure the quality of products, specific tests for shampoo formulations including the determination of dry residue and moisture content, total surfactant activity, salt content, surface tension, detergency tests were carried out. 


\subsection{Physical Appearance/Visual Inspection}

The formulations prepared were evaluated in terms of their clarity, foam producing ability and fluidity.

\subsection{Determination of $\mathrm{pH}$}

The $\mathrm{pH}$ of $10 \%$ shampoo solution in distilled water was determined at room temperature $25^{\circ} \mathrm{C}$.

\subsection{Dete rmine Percent Of Solids Contents}

A clean dry evaporating dish was weighed and added 4 grams of shampoo to the evaporating dish. The dish and shampoo was weighed. The exact weight of the shampoo was calculated only and put the evaporating dish with shampoo was placed on the hot plate until the liquid portion was evaporated. The weight of the shampoo only (solids) after drying was calculated.

\subsection{Surface Tension Measurement}

Measurements were carried out with a $10 \%$ shampoo dilution in distilled water at room temperature. Thoroughly clean the stalagmometer using chronic acid and purified water. Because surface tension is highly affected with grease or other lubricants 5,6 . The data calculated by following equation given bellow: where W1 is weight of empty beaker. W2 is weight of beaker with distilled water. W3 is Weight of beaker with shampoo solution. N1 is no. of drops of distilled water. N2 is no. of drops of shampoo solution. R1 is surface tension of distilled water at room temperature. R2 is surface tension of shampoo solution.

\subsection{Dirt dispersion}

Two drops of shampoo were added in a large test tube contain $10 \mathrm{ml}$ of distilled water. 1 drop of India ink was added; the test tube was stoppered and shakes it ten times. The amount of ink in the foam was estimated as None, Light, Moderate, or Heavy.

\subsection{Rheological Evaluations}

The viscosity of the shampoos was determined by using Brookfield Viscometer (Model DV-l Plus, LV, USA) set at different spindle speeds from 0.3 to $10 \mathrm{rpm} 3$. The viscosity of the shampoos was measured by using spindle T95. The temperature and sample container's size was kept constants during the study.

\subsection{Foaming Ability and Foam Stability}

Cylinder shake method was used for determining foaming ability. $50 \mathrm{ml}$ of the $1 \%$ shampoo solution was put into a $250 \mathrm{ml}$ graduated cylinder and covered the cylinder with hand and shaken for 10 times. The total volumes of the foam contents after 1 minute shaking were recorded. The foam volume was calculated only. Immediately after shaking the volume of foam at 1 minute intervals for 4 minutes were recorded.

\section{RES ULTS AND DIS CUSSION}

In the present Research work Total 5 Formulations were made and evaluated for different parameters. Physical Appearance/Visual Inspection, the results of visual inspection of series of formulations are listed in Table 2. As can be seen, all formulations had the good characteristics with respect to foaming. The $\mathrm{pH}$ of shampoos has been shown to be important for improving and enhancing the qualities of hair, minimizing irritation to the eyes and stabilizing the ecological balance of the scalp10. The current trend to promote shampoos of lower $\mathrm{pH}$ is one of the ways to minimize damage to the hair. Mild acidity prevents swelling and promotes tightening of the scales, there by inducing shine. As seen from Table 1 , all the shampoos were acid balanced and were ranged 5.5 to 5.9 , which is near to the skin $\mathrm{p}^{\mathrm{h}}$. Percent of Solids Contents If the shampoo has too many solids it will be hard to work into the hair or too hard to wash out. The result of percent of solids contents is tabulated in table 1, and was found between 22$29 \%$. As a result, they were easy to wash out. Dirt Dispersion Shampoo that cause the ink to concentrate in the foam is considered poor quality, the dirt should stay in water. Dirt that stays in the foam will be difficult to rinse away. It will redeposit on the hair. All five shampoos showed similar results. These results indicate that no dirt would stays in the foam; so prepared and marketed formulations are satisfactory. Surface tension measurement it has been mentioned that a proper shampoo should be able to decrease the surface tension of pure water to about $40 \mathrm{dynes} / \mathrm{cm} 12$. Surface tension reduction is one 
of the mechanisms implicated in detergency. The reduction in surface tension of water from 72.8 dynes/cm to 35.37 dynes/ $\mathrm{cm}$ by the herbal shampoos is an indication of their good detergent action. The results are shown in Table 2.

Table2. Evaluation of Formulation for Physical Appearance, $P^{h}$ and Solids

\begin{tabular}{|c|c|c|c|c|c|c|}
\hline Formulations & Appearance & $\mathbf{p H}$ & Solids (\% ) & $\begin{array}{c}\text { Cleaning } \\
\text { action (\%) })\end{array}$ & $\begin{array}{c}\text { Surface } \\
\text { Tension } \\
(\mathbf{d y} / \mathbf{c m})\end{array}$ & $\begin{array}{c}\text { Dirt } \\
\text { Deposition }\end{array}$ \\
\hline F1 & $\begin{array}{c}\text { Dark Brown, Good } \\
\text { Foaming }\end{array}$ & $5.51 \pm 0.02$ & $22.11 \pm 0.02$ & $24.21 \pm 0.05$ & $32.15 \pm 0.02$ & Light \\
\hline F2 & $\begin{array}{c}\text { Dark Brown, Good } \\
\text { Foaming }\end{array}$ & $5.53 \pm 0.07$ & $24.51 \pm 0.02$ & $32.51 \pm 0.09$ & $33.22 \pm 0.12$ & Light \\
\hline F3 & $\begin{array}{c}\text { Dark Brown, Good } \\
\text { Foaming }\end{array}$ & $5.61 \pm 0.02$ & $29.31 \pm 0.02$ & $18.81 \pm 0.08$ & $31.37 \pm 0.62$ & Light \\
\hline F4 & $\begin{array}{c}\text { Dark Brown, Good } \\
\text { Foaming }\end{array}$ & $5.81 \pm 0.04$ & $25.41 \pm 0.02$ & $33.61 \pm 0.05$ & $34.60 \pm 0.32$ & Light \\
\hline F5 & $\begin{array}{c}\text { Dark Brown, Good } \\
\text { Foaming }\end{array}$ & $5.91 \pm .01$ & $28.21 \pm 0.02$ & $32.11 \pm 0.02$ & $33.61 \pm 0.42$ & None \\
\hline
\end{tabular}

The results of rheological evaluation showed that the viscosity of the samples changes gradually with the increase in rpm, therefore the shampoo formulations were time dependent. Secondly as the data showed the viscosity decreases with increase in rpm, so the shampoo formulations were shear thinning or pseudo plastic in nature. These formulations showed pseudo plastic behavior which is a desirable attribute in shampoos formulation. At low rpm the herbal shampoos showed high viscosity and increase in the shear rate the viscosity of the shampoos drops, this is a favorable property which eases the spreading of the shampoos on hair. The results obtained from the rheological studies were fitted into different flow behaviors, using the linear or non-linear regression. Table 3 shows the goodness of fitting indices for Newtonian, plastic and pseudo plastic flow behaviors. As can be seen in the Table 2, all the formulations followed a pseudo plastic rheogram.

Table3. Evaluation of Formulations for Viscosity

\begin{tabular}{|c|c|c|c|c|c|}
\hline \multirow{2}{*}{ RPM } & \multicolumn{5}{|c|}{ Viscosity(cp) } \\
\cline { 2 - 6 } & F1 & F2 & F3 & F4 & F5 \\
\hline 0.3 & 95733.33 & - & 83433.33 & - & - \\
\hline 0.5 & 82150.00 & 60765.00 & 73583.33 & - & 17533.33 \\
\hline 1.0 & 54150.00 & 42666.66 & 51516.67 & - & 14916.67 \\
\hline 1.5 & 50916.67 & 33350.00 & 40450.00 & - & 11478.33 \\
\hline 2.5 & 38778.33 & 23978.33 & 28851.66 & 7598.33 & 9158.33 \\
\hline 5 & 25425.00 & 14645.33 & 17651.66 & 5945.00 & 7766.66 \\
\hline 10 & 15775.00 & 8541.00 & 10741.66 & 5156.67 & \\
\hline
\end{tabular}

Foaming ability and foam stability although foam generation has little to do with the cleansing ability of shampoos, it is of paramount importance to the consumer and is therefore an important criterion in evaluating shampoos. All the five shampoos showed similar foaming characteristics in distilled water. The foam retention ability of five samples is given in figure 1. All five shampoos showed comparable foaming properties. The foam stability of herbal shampoos is listed in table 4. A point to be noted here is that there does not seem to be any direct correlation between detergency and foaming, which only confirms the fact that a shampoo that foams well need not clean well. The final formulation produced stable foams there was little bet change in foam volume.

Table4. Evaluation of Foam Stability of Herbal Shampoo Formulations

\begin{tabular}{|c|c|c|c|c|c|}
\hline \multirow{2}{*}{ Time in Mins } & \multicolumn{5}{|c|}{ Foam Volume (ml) } \\
\cline { 2 - 6 } & F1 & F2 & F3 & F4 & F5 \\
\hline 1Min & 170 & 180 & 140 & 180 & 180 \\
\hline 2Min & 168 & 177 & 137 & 178 & 177 \\
\hline 3Min & 167 & 175 & 134 & 175 & 174 \\
\hline 4Min & 165 & 174 & 135 & 174 & 172 \\
\hline 5Min & 164 & 173 & 134 & & \\
\hline
\end{tabular}




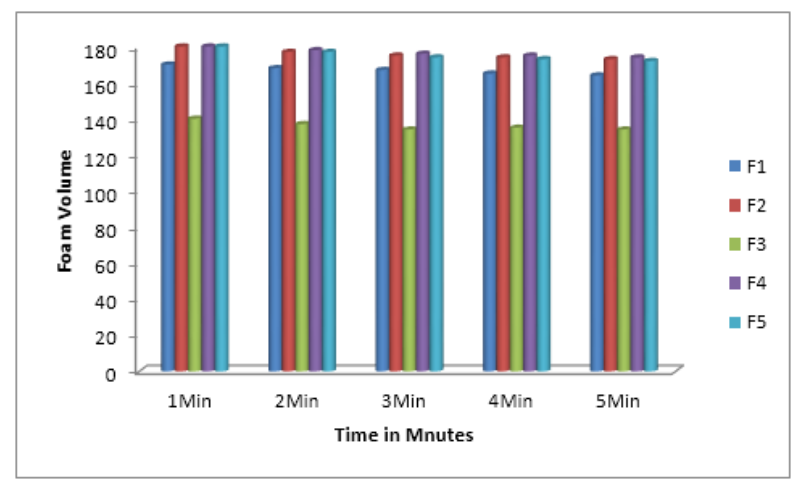

Fig1. Foam Stability of Herbal Shampoos Formulations

\section{CONCLUSION}

The present investigation demonstrated that Bhringraj Powder showed excellent antifungal activity. An antidandruff shampoo was formulated by incorporating Bhringraj Powder as main antidandruff ingredients. As additives play an important role in defining the performance, stability and aesthetic appeal of any formulation, this point was kept in mind while selecting the additives for the shampoo formulation. Finally the antidandruff shampoo was formulated and evaluated. As seen from the results, it is possible to formulate an herbal antidandruff shampoo that is not only equivalent in its performance to the synthetic ones but also have better safety, efficacy and purity.

\section{ACKNOWLEDGEMENT}

The authors are Thankful to Rev Dr. K. V. K. Rao and Dr. P. Venkateswara Rao, principal for providing the facilities to carry out this research work in St. Mary's Group of Institutions Guntur.

\section{REFERENCES}

[1] B.M.Mithal, R.N.Saha, A hand book of cosmetics, first edition, 2000.

[2] Jaya preeti P. Padmini K., Srikanth J, Lohita M, Swetha K Vengal rao p., a review on H Herbal Shampoo and its Evaluation, Asian J. Pharm. Ana. 3(4): 2013; 153-156 3.

[3] A.R.Mainkar, C.I.Jolly, Formulation of natural shampoos, International journal of cosmetic science, 2001, 223, 59-62.

[4] Ronni wolf, MD, Danny wolf, MD, Soaps, Shampoos and Detergents, Clinics in Dermatology, 2001; 19:393-397.

[5] Anusha. P. Harish. G.B.Pragathi Kumar, Formulation and evaluation of herbal anti-dandruff shampoo. IJRPB 1(6), 2013, 835-839.

[6] Aghel N., Moghimipour B. and Dana R.A., Iranian Journal of Pharmaceutical Research (2007) 6(3), $167-$ 172.

[7] Chandrani D, Lubaina SZ and Soosamma M, A review of antifungal effect of plant extract vs. chemical substances against malassezia spp., Int J Pharm Bio Sci, 3(3), 2012, 773 - 780.

[8] Mansuang Wuthi-udomlert, Ployphand Chotipatoomwan, Sasikan Panyadee and Wandee Gritsanapan, Inhibitory effect of formulated lemongrass shampoo on Malassezia furfur: a yeast Associated with dandruff, Southeast Asian j trop med public health, 42(2), 2011, 363-369.

[9] Naveen S, Karthika S, Sentila R, Mahenthiran R, Michael A, In-vitro evaluation of herbal and chemical agents in the management of Dandruff, J. Microbiol. Biotech. Res., 2 (6), 2012, 916-921.

[10] Richa Madhu Sharma, Kinjal Shah, Janki Patel, Evaluation of Prepared Herbal Shampoo Formulations and To Compare Formulated Shampoo with Marketed Shampoos, Int J Pharm Pharm Sci, 3(4), 2011, 402-405.

[11] Singla chhavi, Drabu sushma, Ali Mohammed, Potential of herbals as an antidandruff agents, 2(3), 2011, 16-18.

Citation P. Vinod Kumar, et al., "Formulation and Evaluation ofHerbal Anti-Dandruff Shampoo from Bhringraj Leaves", ARC Journal of Pharmaceutical Sciences (AJPS), vol. 4, no. 2, p. 29-33, 2018. http://dx.doi.org/10.20 431/2455-1538.0402005

Copyright: () 2018 Authors. This is an open-access article distributed under the terms of the Creative Commons Attribution License, which permits unrestricted use, distribution, and reproduction in any medium, provided the original author and source are credited 